Liu, H.-H., \& Colman, A. M. (2009). Ambiguity aversion in the long run: Repeated decisions under risk and uncertainty. Journal of Economic Psychology, 30, 277-284.

\title{
Ambiguity aversion in the long run: Repeated decisions under risk and uncertainty
} Hsin-Hsien Liu ${ }^{\mathrm{a}, *}$, Andrew M. Colman ${ }^{\mathrm{b}}$

a Department of Marketing and Logistics, Southern Taiwan University, 1 Nantai Street, Yung-Kang, Tainan 710, Taiwan

${ }^{\mathrm{b}}$ School of Psychology, University of Leicester, Leicester LE1 7RH, UK

\begin{abstract}
In two experiments, decision makers chose between risky and ambiguous gambles under conditions of both single (unrepeated) and multiply repeated choices. The gambles were presented either as modified Ellsberg urn choices or as marketing strategy decisions. In both experiments, decision makers chose the ambiguous options more frequently in the repeated-choice than the single-choice conditions. More decision makers made risky single choices and ambiguous repeated choices than ambiguous single choices and risky repeated choices. Decision makers expressed more self-rated confidence in their repeated than their single ambiguous choices. These findings are interpreted in the light of findings on repeated decision making under risk and theories of loss aversion and ambiguity aversion.
\end{abstract}

JEL Classification: C91; D81; M31

PsycINFO Classification: 2340; 3900; 3920

Keywords: Ambiguity aversion; Ellsberg paradox; Loss aversion; Repeated decisions; Risk aversion

\section{Introduction}

Decisions with uncertain outcomes are often made repeatedly. For example, gamblers play the same games repeatedly, savers choose repeatedly where to invest their money, and chess players choose repeatedly from their opening repertoires. A remarkable feature of such repeated decisions is that people tend to approach them in a strikingly different frame of mind from the way they approach single or unrepeated decisions. The classic illustration of this is the following gamble that Samuelson (1963) offered to a colleague: on the basis of a coin toss, a 50\% chance of winning \$200 and a 50\% chance of losing \$100. The colleague declined the gamble and commented: “I won’t bet because I would feel the \$100 loss more than the \$200 gain. But I'll take you on if you promise to let me make 100 such bets" (p. 109).

Although this argument seems intuitively appealing, Samuelson (1963) labeled it a fallacy and proved a theorem to the effect that "if you would always refuse to take favorable odds on a single toss, you must rationally refuse to participate in any (finite) sequence of such tosses" (p. 111). Informally, assume that after 99 gambles a decision maker were offered the 100th. 
By assumption, a single gamble is unacceptable, hence this one would be declined.

Furthermore, knowing that the 100th gamble would be declined, the decision maker would have recognized the 99th to be, in effect, a single gamble, and would therefore have declined it previously. The same reasoning unfolds back to the first decision, which would also have been declined. Tversky and Bar-Hillel (1983) proved that declining the single gamble and accepting the repeated gamble violates unobjectionable conditions of transitivity or dominance.

Psychologically, the gamble repeated 100 times nevertheless seems more appealing than the single gamble. It offers an expected gain of $\$ 5,000$ with only a tiny $(<1 \%)$ probability of loss, compared with a $50 \%$ probability of loss from the single gamble. Lopes (1981) endorsed the policy of accepting only the repeated gamble and concluded from Samuelson's (1963) example that expected utility maximization "is simply not sensible" (p. 385). Many years later, she wrote that she would "neither repent nor recant" that conclusion (Lopes, 1996, p. 183).

Experimental evidence suggests that human decision makers share similar intuitions. Montgomery and Adelbratt (1982) explained the concept of expected value to their participants, provided them with sets of 17 gambles, each displayed with its expected value, and invited them to select a preferred option from each set. The participants usually deviated from expected value when making single choices - some even remarking that numerical calculation was irrelevant to gambles played only once - but maximized expected value when repetitions were allowed. Most of Redelmeier and Tversky's (1992) participants accepted repeated gambles but rejected the corresponding single gamble. DeKay and Kim (2005) replicated this effect, at least for decisions involving monetary payoffs. Keren and Wagenaar (1987) and Keren (1991) observed violations of expected utility theory (certainty and possibility effects) in single choices but not in repeated choices. Wedell and Böckenholt (1990, 1994) reported that preference reversal, another violation of expected utility theory, was significantly reduced under repeated choices, and most participants justified their repeated but not their single choices by integrating probabilities with outcomes. Experimental evidence converges on the conclusion that decision makers generally conform more closely to normative expected utility theory in repeated than single gambles.

\subsection{Loss aversion}

A compelling reason for rejecting a single gamble with positive monetary value is loss aversion. This is a greater sensitivity to losses than to gains, represented in prospect theory (Kahneman \& Tversky, 1979) by a point of inflection in its utility function at the origin. Recall that Samuelson's (1963) colleague refused the single gamble for this very reason. Tversky and Kahneman (1992) estimated that losses are weighted 2.25 times as heavily as gains, relative to the status quo point, so that the disutility of losing $\$ 100$ is more than twice the utility of gaining $\$ 100$. Suppose a decision maker's utility for gains is equal to monetary 
value but for losses is equal to monetary value multiplied by 2.25 . Then the expected utility of the single gamble offering 50\% chance of winning $\$ 200$ and a $50 \%$ chance of losing $\$ 100$ is negative: $(1 / 2)(200)+(1 / 2)(-100 \times 2.25)=-25$, but the expected utility of the repeated version is positive. For example, the expected utility of a portfolio of two gambles is $(1 / 4)(400)+(1 / 2)(100)+(1 / 4)(-200 \times 2.25)=37.50$. This provides a possible explanation for the greater attractiveness of repeated gambles. When loss aversion arises from narrow framing, it is called myopic loss aversion (Benartzi \& Thaler, 1995, 1999; Thaler, Tversky, Kahneman, \& Schwartz, 1997). For example, myopic loss aversion could cause Samuelson's colleague to decline a single gamble offered on 100 successive days, although the 100-gamble sequence would be acceptable if broadly framed as an aggregated portfolio.

In mixed gambles involving potential gains and losses, an outcome parameter of particular salience to loss-averse decision makers is the probability of loss. Preferences for gambles are known to be more highly correlated with probabilities of gain/loss than with payoff sizes (Lichtenstein \& Slovic, 1971; Slovic \& Lichtenstein, 1968; Tversky, Sattath, \& Slovic, 1988). In many cases, the probability of loss is smaller in a repeated gamble than in the corresponding single gamble, although this is counterbalanced by the increased size of the possible loss. Loss aversion coupled with a tendency to focus specifically on the probability of loss may explain the relative attractiveness of such repeated gambles.

For practical and ethical reasons, experiments incorporating actual rather than hypothetical payoffs generally involve smaller or larger gains, rather than gains and losses, ruling out direct investigation of loss aversion and the risk-seeking behavior that it is believed to induce. However, Bosch-Domènech and Silvestre (2006) studied actual gains and losses and found that, for large losses, most decision makers were risk averse rather than risk seeking. Nevertheless, in circumstances in which loss aversion does occur, it seems reasonable to assume that it extends, to a degree at least, to opportunity costs. A loss-averse decision maker is likely to be motivated not only to avoid absolute losses, but also to avoid relative losses involved in forgoing potentially more rewarding options.

\subsection{Ambiguity aversion}

Repetition effects have been investigated thoroughly in known-risk gambles, but no systematic comparisons of single and repeated ambiguous gambles appear to have been published, although Einhorn and Hogarth (1986) included a parameter representing the amount of new information gained (e.g., from repetitions) in their formal model of decision making under ambiguity (Equation 9; see also Einhorn \& Hogarth, 1985, Equations 8 and 9). Ambiguous choices are those for which outcome probabilities cannot be calculated or estimated from empirical evidence. Knight (1921), who first highlighted them, used the term uncertain for what is less ambiguously called ambiguous and gave an example of two men drawing balls from an urn: "One man knows that there are red and black balls, but is ignorant of 
the numbers of each; another knows that the numbers are three of the former to one of the latter" (pp. 218-219). The first man chooses under ambiguity; the second under risk.

Most decision makers prefer risky to ambiguous choices. This is the ambiguity aversion effect, the classic demonstration of which is the Ellsberg paradox (Ellsberg, 1961). Two urns are filled with red and black balls, Urn $A$ containing 50 red and 50 black balls, randomly mixed, and Urn $B$ an unknown ratio of 100 red and black balls, randomly mixed. A decision maker chooses a color (red or black) and then an urn ( $A$ or $B)$ from which to draw, winning a prize for drawing a ball of the chosen color. Typically, 60-70\% of decision makers prefer to draw from the risky Urn $A$, and experimental evidence has shown this effect to be powerful and robust (Camerer \& Weber, 1992; Curley \& Yates, 1989; Frisch \& Baron, 1988; Keren \& Gerritsen, 1999; Pulford \& Colman, 2008; Rode, Cosmides, Hell, \& Tooby, 1999). It occurs even when decision makers, though ignorant of the ratio of red to black balls in $\operatorname{Urn} B$, know that every possible ratio is equally likely, although this second-order probability information implies that the first-order probabilities are equal.

\subsection{Modified Ellsberg urn choice}

In the experiments described below, we used a modified Ellsberg urn choice inspired by Samuelson's (1963) asymmetric gamble. For a risky choice, the payoff for success was NT 500 (US\$16.50), and for an ambiguous choice, it was NT 600 (US\$20.00). Assuming all possible distributions to be equally likely, an expected payoff maximizer should prefer the ambiguous choice. Furthermore, a decision maker is more likely to receive a larger payoff from the ambiguous than the risky choice. Comparing a risky and an ambiguous pair of options $(R, A)$, there are four equiprobable combinations: $(0,0),(0,600),(500,0)$, and $(500,600)$. In two cases the ambiguous option $A$ pays more than the risky option $R$, and in one case the risky option pays more. A decision maker is therefore twice as likely to gain more from the ambiguous option $(p=1 / 2)$ than from the risky option $(p=1 / 4)$.

The expected value of the risky option repeated 100 times is $100 \times 1 / 2 \times 500=$ NT 25,000. The probability of gaining more by choosing the repeated ambiguous option is calculated as follows. If every possible distribution is equally likely, then, by symmetry, the probability of success on each component gamble is $1 / 2$. A decision maker gains more from 100 repetitions of the ambiguous option than from 100 repetitions of the risky option (expected value $=\mathrm{NT}$ 25,000) if $s$ ambiguous gambles are successful and 600s > NT 25,000. This holds for $s>$ $25,000 / 600$, that is, for all $s \geq 42$. The probability $q$ of obtaining at least 42 successes in 100 gambles is

$$
q=\sum_{s=42}^{100}\left(\begin{array}{c}
100 \\
s
\end{array}\right) .5^{s} .5^{100-s}=.96
$$


The probability of gaining more from the ambiguous than the risky option is therefore $p=.50$ for a single choice and $q=.96$ for a choice repeated 100 times. A decision maker should accordingly prefer the repeated ambiguous choice to the single ambiguous choice.

The rest of this article is structured as follows. In Section 2 we report an experiment comparing single and repeated risky and ambiguous choices, framed either as modified Ellsberg urn choices or as marketing strategy choices. In Section 3 we report a replication in which we counterbalance presentation orders and provide participants with second-order probabilities for ambiguous options. Section 4 contains a general discussion.

\section{Experiment 1}

The purpose of this experiment was to explore, apparently for the first time, decision making in single and repeated choices under both risk and ambiguity. Our main hypothesis was that decision makers would choose ambiguous options more frequently in repeated than single choices, repetition thus dampening ambiguity aversion. To provide an indication of the robustness of any repetition effects, we presented the decisions as either a modified Ellsberg urn choice or a marketing strategy choice. We did not attempt to make the marketing strategy choice exactly equivalent to the urn choice, because it was not part of our intention to compare the two; we needed only to compare single and repeated choices separately in each.

We also collected self-ratings of decision confidence. Pulford and Colman (2007, 2008) suggested that ambiguity aversion may be associated with intolerance of uncertainty. They cited evidence that most people prefer to avoid events and circumstances that are difficult to understand, and they interpreted ambiguity aversion as a particular manifestation of this. A subsidiary hypothesis was accordingly that participants would be more confident of repeated than single ambiguous choices.

\subsection{Experimental design and decision tasks}

Each participant made a single urn choice followed by a repeated urn choice or a single followed by a repeated marketing strategy choice. The experimental design was a $2 \times 2$ factorial experiment: Repetition (single versus repeated) $\times$ Frame (urn choice versus marketing strategy choice), with repeated measures on the repetition factor. The choices were presented to the participants as follows (translated from Mandarin).

Single urn choice. Image that you have a ticket to participate in one of two bets: Urn 1 contains 50 red balls and 50 green balls, whereas Urn 2 contains 100 red and green balls in unknown proportions. The proportion of red and green balls in Urn 2 is governed by computer. Draw a ball blindly from an urn and guess its color. If you choose Urn 1 and your guess is correct, then you win NT 500. If you choose Urn 2 and your guess is correct, then you win NT 600. Which urn would you choose? 
Repeated urn choice. If such gamble is repeated 100 times, then once you choose the urn, you have to play the bet 100 times with the same urn. Once you have decided how to play, which urn would you choose? If you choose Urn 1, then you have to guess the color each time before you draw a ball, and you will win NT 500 each time your guess is right or nothing if your guess is wrong. If you choose Urn 2, then the computer resets the balance of red and green balls each time before you guess the color and draw a ball. You will win NT 600 each time your guess is right or nothing if your guess is wrong. The total money you win depends on the number of times you win and the prize (NT 500 if you choose Urn 1 and NT 600 if you choose Urn 2, each time).

Single marketing strategy choice. Image that you are the CEO of an international company, and the marketing manager has recently planned two new service programs for Taiwanese customers based on the company's advantages: M program and Z program. You believe that both programs might create advantages and bring huge profits for the company; however, you have to choose one of them rather than both because of your limited resources. Based on previous experience and the ability of competitors, the advisors of your company all agree that the success probability of Option M would be $50 \%$. However, they argue that the probability of success of Option $\mathrm{Z}$ might be from $40 \%$ to $80 \%$, because they are unsure whether competitors will be able to offer the same service. If the program you choose is successful, then no matter whether you choose $\mathrm{M}$ or $\mathrm{Z}$, you would make NT 50 million for your company. Which program would you choose?

Repeated marketing strategy choice. As the CEO, you find out that 10 other overseas subsidiary companies face the same choices between the two service programs. The market scales of the 10 countries in which your subsidiary companies are located are similar to that of Taiwan. Because your company is international, you hope to keep the service programs in all subsidiaries identical. Now you have to decide which program will be conducted (the success or failure of the program in one country will not influence the probability of success or failure in the other country). Which one would you choose?

\subsection{Participants}

A convenience sample of 138 students ( 80 women and 58 men, mean age 24.25 years), recruited by approaching students on two different campuses in Taiwan, were assigned randomly to urn choice or marketing strategy choice treatment conditions, each containing 69 participants. Participants made decisions involving hypothetical payoffs but were not remunerated personally.

\subsection{Procedure}

Participants read a page of instructions and then indicated their preferred options, first for the single choice, and then, on a separate page, for the repeated choice. In the urn choice 
condition, they responded to the following question in writing after each choice: "On the whole, you feel _ \% confident that your decision would bring you the most profit." In the marketing strategy choice condition, the question was: "On the whole, you feel _ \% confident that your decision would bring most profit for your company." Finally, they were thanked and debriefed.

\subsection{Results and discussion}

The results are shown in Table 1. Because single and repeated choices were not independent (the same participants made both), we performed chi-square goodness of fit tests separately for single and repeated choices. In the single urn choice, $31.88 \%(22 / 69)$ of participants chose the ambiguous option, $\chi^{2}(1)=9.06, p<.01, w=.36$ (medium ambiguity aversion effect size), rising to $50.72 \%$ (35/69) for the repeated choice: $\chi^{2}(1)=0.01$, ns (no ambiguity aversion). Of the participants who chose the risky single urn option, $38.30 \%$ (18/47) switched to the ambiguous repeated option, whereas of those who chose the ambiguous single option, only $22.73 \%$ (5/22) switched to the risky repeated option. Application of the CATMOD modeling technique for repeated-measures categorical data (Stokes, Davis, \& Koch, 1995) shows the difference between these two proportions to be significant: $\chi^{2}(1)=8.24, p<0.01, w=.35$ (medium). These choice data confirm our main hypothesis for urn choices.

\section{Table 1}

Experiment 1: Numbers of participants who chose risky $(R)$ and ambiguous $(A)$ options in single and repeated choices, and differences in self-rated confidence (DiffConf) between repeated and single choices for participants who chose ambiguous repeated options

\begin{tabular}{|c|c|c|c|c|c|c|c|c|}
\hline \multirow[b]{3}{*}{ Single choice } & \multicolumn{8}{|c|}{ Repeated choice } \\
\hline & \multicolumn{3}{|c|}{ Urn choice } & \multicolumn{5}{|c|}{ Marketing strategy choice } \\
\hline & $R$ & $A$ & Total & DiffConf (\%) & $R$ & $A$ & Total & DiffConf (\%) \\
\hline$R$ & 29 & 18 & 47 & 14.45 & 13 & 17 & 30 & 10.89 \\
\hline$A$ & 5 & 17 & 22 & 6.24 & 7 & 32 & 39 & 4.69 \\
\hline Total & 34 & 35 & 69 & & 20 & 49 & 69 & \\
\hline
\end{tabular}

In the marketing strategy condition, $56.52 \%(39 / 69)$ of participants chose the ambiguous single option, $\chi^{2}(1)=1.17, n s$, rising to $71.01 \%(49 / 69)$ in the repeated choice: $\chi^{2}(1)=12.19$, $p<.001, w=.42$ (medium to large). The absence of ambiguity aversion in the single choice may be due to the smaller range of probabilities of success for the ambiguous marketing strategy choice (range 40-80\%) than the ambiguous urn choice (range 0-100\%), probably diminishing the effect of ambiguity aversion (MacCrimmon \& Larsson, 1979; Yates \& Zukowski, 1976), but a repetition effect is nonetheless evident. Furthermore, 56.67\% (17/30) 
of participants who chose the risky single option switched to the ambiguous repeated option, compared to only $17.95 \%$ (7/39) who chose the ambiguous single option and switched to the risky repeated option, and CATMOD analysis shows the difference between these proportions to be significant: $\chi^{2}(1)=4.43, p=0.03, w=.25$ (small to medium). These data confirm our main hypothesis for marketing strategy choices.

Participants who chose ambiguous repeated options were more confident of those choices than of their single choices. In Table 1, the columns labeled "DiffConf" show differences between self-rated confidence in repeated and single choices for participants who chose ambiguous repeated options. Larger percentages indicate greater increases in self-rated confidence from single (risky or ambiguous) choices to repeated ambiguous choices. For participants who chose the risky single urn option, then the ambiguous repeated option, self-rated confidence increased from $55.83 \%$ to $70.28 \%$, and this difference (14.45\%) is significantly different from zero according to a one-sample $t$ test: $t(17)=3.73, p<.01, d=$ 0.88 (large). Similarly, for those who chose the ambiguous single urn option, then the ambiguous repeated option, self-rated confidence increased significantly from $65.29 \%$ to $71.53 \%: t(16)=4.45, p<.01, d=1.08$ (large). In contrast, participants who chose the risky repeated urn option (omitted from Table 1) did not show significant changes in confidence relative to risky single choices $t(28)=1.22, n s$, or ambiguous single choices, $t(4)=-1.63, p$ $<.10, n s$. These findings confirm our subsidiary hypothesis for urn choices.

In the repeated marketing strategy choice condition, self-rated confidence of participants who chose the risky single option increased from $61.76 \%$ to $72.65 \%$ when they switched to the ambiguous repeated option, $t(16)=3.82, p<.01, d=0.93$ (large); and confidence of those who chose the ambiguous single option increased from $66.72 \%$ to $71.41 \%$ when they chose the ambiguous repeated option: $t(31)=3.26, p<.01, d=0.58$ (medium). In contrast, those who chose the risky repeated option (omitted from Table 1) showed no significant changes in confidence relative to their previous risky single choices, $t(12)=-1.24$, $n s$, or ambiguous single choices $t(6)=-1.10, n s$. These confidence data replicate those for urn choices and confirm our subsidiary hypothesis for marketing strategy choices.

\section{Experiment 2}

Two potentially important extraneous variables were uncontrolled in Experiment 1. First, the higher expected values associated with ambiguous than risky options relied on participants assuming that all ratios of successful to unsuccessful outcomes in the ambiguous choices were equally likely, but this was not made explicit to them. Second, the presentation orders of the single and repeated choices were not counterbalanced, and consequently differences between single and repeated choices could conceivably have been due to an order effect. Experiment 2 was designed to correct these imperfections. 


\subsection{Participants}

A convenience sample of 243 participants (180 women and 63 men, mean age 21.95 years), was recruited by approaching students on two different campuses in Taiwan. Participants were assigned randomly to four experimental conditions: single then repeated urn choice $(N=63)$, repeated then single urn choice $(N=55)$, single then repeated marketing strategy choice $(N=63)$, and repeated then single marketing strategy choice $(N=62)$. As in Experiment 1, participants were not remunerated.

\subsection{Design and procedure}

The design and procedure followed Experiment 1, with two exceptions. First, the presentation orders of single and repeated choices were counterbalanced, with appropriate modification of the question requesting second choices. Second, participants were told explicitly that every possible ratio of successful to unsuccessful outcomes of ambiguous choices was equally likely. In the urn choice condition, the instructions contained the following sentence (in Mandarin), the words in italics having been added for this experiment: "The proportions of red and green balls in Urn 2 are governed by computer, and every possible ratio is equally likely (i.e., the probabilities of $0,1,2, \ldots, 100$ red balls are equally likely)." In the marketing strategy choice condition, the corresponding sentence was: "However, they argue the success probability of Option Z might be from 40-80\% (because they are unsure whether competitors will be able to offer the same service), but they believe that every possible probability of success is equally likely."

The experimental design was $2 \times 2 \times 2$ factorial: Frame (urn choice versus marketing strategy choice $) \times$ Order $($ single choice first versus repeated choice first $) \times$ Repetition $($ single versus repeated), with repeated measures on the repetition factor only.

\subsection{Results}

CATMOD analysis, with risky or ambiguous urn choice as the dependent variable and order, repetition, and the Order $\times$ Repetition interaction as sources of variance, revealed that only the switch in participants' preferences between single and repeated choices was significant, $\chi^{2}(1)=6.39, p=.01, w=.16$ (small). We therefore pooled the data across the two task orders for further analysis (see Table 2). 
Table 2

Experiment 2: Numbers of participants who chose risky $(R)$ and ambiguous $(A)$ options in single and repeated choices, and differences in self-rated confidence (DiffConf) between repeated and single choices for participants who chose ambiguous repeated options

\begin{tabular}{ccccccccc}
\hline & \multicolumn{1}{c}{ Urn choice } & \multicolumn{5}{c}{ Marketing strategy choice } \\
\cline { 2 - 9 } Single choice & $R$ & $A$ & Total & DiffConf $(\%)$ & $R$ & $A$ & Total & DiffConf $(\%)$ \\
\hline \multirow{nyyyyyyyy}{*}{$R$} & 39 & 34 & 73 & 7.38 & 30 & 48 & 78 & $11.14 \%$ \\
$A$ & 16 & 29 & 45 & 9.52 & 10 & 37 & 47 & $5.68 \%$ \\
Total & 55 & 63 & 118 & & 40 & 85 & 125 & \\
\hline
\end{tabular}

For urn choices, only $38.14 \%$ (45/118) of participants chose the ambiguous single option, $\chi^{2}(1)=6.64, p<.01, w=.24$ (medium ambiguity aversion effect), rising to $53.39 \%(63 / 118)$ for the repeated option: $\chi^{2}(1)=0.54, n s$. Moreover, $46.57 \%(34 / 73)$ of participants who chose the risky single option switched to the ambiguous repeated option, whereas only $35.56 \%$ (16/45) of those who chose the ambiguous single option switched to the risky repeated option, and CATMOD analysis shows the difference between these proportions to be significant: $\chi^{2}(1)$ $=6.39, p=.01, w=.23$ (small to medium). These results replicate those of Experiment 1 .

For marketing strategy choices, $37.60 \%$ (47/125) of participants chose the ambiguous single option, $\chi^{2}(1)=7.69, p<.01, w=.25$ (small to medium ambiguity aversion effect), rising to $68 \%(85 / 125)$ in the repeated choice: $\chi^{2}(1)=16.20, p<.001, w=.36$ (medium to large). Moreover, $61.54 \%$ (48/78) of participants who chose the risky single option switched to the ambiguous repeated option, whereas only $21.28 \%$ (10/47) of those who chose the ambiguous single option switched to the risky repeated option, and CATMOD analysis shows that the difference between these proportions is significant: $\chi^{2}(1)=31.17, p<0.01, w=.50$ (large). These findings on switching also replicate those of Experiment 1.

In the urn choice condition, order had no significant effect on self-rated confidence, $F(1$, $116)=0.37, n s$, therefore we pooled the confidence data across orders. As in Experiment 1, self-rated confidence of participants who chose the risky option in the single urn choice was significantly higher when they chose the ambiguous repeated option: $50.41 \%$ versus $57.79 \%$, $t(33)=1.91, p<.05$, one-tailed, $d=0.33$ (small to medium). Similarly, confidence of participants who chose the ambiguous single option increased significantly when they chose the ambiguous repeated option, from $55.17 \%$ to $64.69 \%: t(28)=3.35, p<.05, d=0.62$ (medium to large). In contrast, confidence hardly differed between single and repeated urn choices for participants who chose the risky repeated option, regardless of whether they chose the risky single option, $t(38)=0.16, n s$, or the ambiguous single option, $t(15)=0.41, n s$. These results corroborate the findings on confidence of urn choices of Experiment 1. 
In the marketing strategy choice condition, because order had no effect on confidence, $F(1,123)=0.00, n s$, we pooled confidence data across orders once again. Confidence of participants who chose the risky option increased when they chose the ambiguous repeated option, from $56.71 \%$ to $67.85 \%: t(47)=4.31, p<.05, d=0.62$ (medium to large). Similarly, participants who chose the ambiguous single option showed higher confidence when they chose the ambiguous repeated option: $65.00 \%$ versus $70.68 \%, t(36)=3.10, p<.05, d=0.51$ (medium). Once again, confidence levels of participants who chose the risky repeated option showed no such differences, regardless of whether they chose the risky single option, $t(29)$ $=-0.49, n s$, or the ambiguous single option, $t(9)=0.83, n s$. These findings relating to confidence of marketing strategy choices also replicate those of Experiment 1.

Taken together, the result of this experiment fully corroborate those of Experiment 1 . The more rigorous controls in this experiment reinforce our confidence in the findings.

\section{General discussion}

Our experiments provide remarkably consistent and robust evidence of repetition effects in decision making under ambiguity. ${ }^{1}$ In particular, participants chose ambiguous options significantly more frequently in repeated-choice than single-choice conditions. This occurred in Experiment 1, when the ambiguous option was truly ambiguous, and in Experiment 2, when the participants knew that all possible probabilities of success of ambiguous choices were equally likely. Our results are broadly consistent with findings on decision making under risk, in which participants have conformed more closely to the normative prescriptions of expected utility theory in repeated than single choices (DeKay \& Kim, 2005; Keren, 1991; Keren \& Wagenaar, 1987; Montgomery \& Adelbratt, 1982; Redelmeier \& Tversky, 1992; Wedell \& Böckenholt, 1990, 1994).

In our single urn choice conditions, significant ambiguity aversion occurred, in line with evidence stretching back to Ellsberg (1961), but in repeated urn choices, a majority of participants chose the ambiguous options in both experiments. Furthermore, significantly more participants chose the risky option in the single urn choice and the ambiguous option in the repeated choice than the other way round, and this result was also replicated even when the orders of single and repeated choices were counterbalanced in Experiment 2.

To provide an indicative check of robustness, both experiments included a marketing strategy choice condition in which some features of the modified Ellsberg urn choice were framed in a lifelike scenario. The urn choice and marketing strategy choice were not designed to be fully equivalent, because we were concerned not with comparing them but with investigating repetition effects separately in each. The number of repetitions, the range of probabilities of success in the ambiguous option, and the payoff structure differed slightly between the urn choices and marketing strategy choices, but these differences have no bearing on the repetition effects that we investigated. In Experiment 1, ambiguity aversion 
did not occur in single marketing strategy choices, probably because of the relatively restricted variance in the probability of success of the ambiguous choice; in Experiment 2, a small to medium ambiguity aversion effect occurred. However, in both experiments, participants chose ambiguous options more frequently in repeated than single market strategy choices, and more participants chose risky single choices and ambiguous repeated choices than the other way round, replicating the asymmetric switching effects found in urn choices.

Taken together, these results suggest that repetition diminishes the effect of ambiguity aversion. Participants found ambiguous options more attractive under conditions of repeated choice. An obvious explanation for this is loss aversion, which has been used to explain decision makers' greater adherence to normative principles in repeated risky gambles (Tversky \& Bar-Hillel, 1983; Thaler et al., 1997). Loss-averse decision makers, especially those who focus on the probability of loss, may be motivated to reject a single ambiguous gamble but to accept the same gamble if it is multiply repeated.

Another possible interpretation is suggested by the comments of some of Montgomery and Adelbratt's (1982) participants that numerical calculation is inapplicable to single gambles. Many people evidently believe that probability is something that applies only in the long run and is irrelevant, or less relevant, to single choices. Such people may feel that the uncertainty inherent in ambiguous choices is somehow eliminated or reduced if the choices are repeated, making probabilities seem more relevant.

Indirect support for this interpretation comes from self-ratings of confidence in our experiments. Participants who chose the ambiguous repeated options were invariably more confident of their choices than of their single choices, whether their single choices were risky or ambiguous. This suggests that the disturbing quality of an ambiguous choice may be dissipated when the choice is repeated. Ambiguity-averse decision makers who feel that a single ambiguous choice is bound to go against them may anticipate a "fairer" outcome from a long sequence of ambiguous choices. People who believe that luck tends to be loaded against them in single events may feel safer in repeated sequences of events, perhaps in the belief that good and bad luck tend to balance out in the long run.

One obvious avenue for further research relates to mixed ambiguous gambles involving potential losses as well as gains. There is evidence suggesting that decision makers tend to choose ambiguous options more frequently under such conditions (Cohen, Jaffray, \& Said, 1985; Goldsmith \& Sahlin, 1983). If loss aversion is a key factor driving repetition effects, then these effects should be sharpened in mixed gambles, because real losses should have stronger psychological effects than mere opportunity costs. Experiments exposing participants to the possibility of losing some of their own money pose serious methodological problems, but gambles involving hypothetical losses or real losses from initial endowments of "house money" are feasible. 


\section{Acknowledgments}

The research reported in this article was supported by research NSC 97-2410-H-218-025 from the National Science Council of Taiwan. The authors are grateful for helpful suggestions from Robin Hogarth, Briony Pulford, and anonymous reviewers.

\section{References}

Benartzi, S., \& Thaler, R. H. (1995). Myopic loss aversion and the equity premium puzzle. Quarterly Journal of Economics, 110, 73-92.

Benartzi, S., \& Thaler, R. A. (1999). Risk aversion or myopia? Choices in repeated gambles and retirement investments. Management Science, 45, 364-381.

Bosch-Domènech, A., \& Silvestre, J. (2006). Reflections on gains and losses: A $2 \times 2 \times 7$ experiment. Journal of Risk and Uncertainty, 33, 217-235.

Camerer, C. F., \& Weber, M. (1992). Recent developments in modeling preference: Uncertainty and ambiguity. Journal of Risk and Uncertainty, 5, 325-370.

Cohen, M., Jaffray, Y., \& Said, T. (1985). Individual behavior under risk and under uncertainty: An experimental study. Theory and Decision, 18, 203-228.

Curley, S. P., \& Yates, J. F. (1989). An empirical evaluation of descriptive models of ambiguity reactions in choice situations. Journal of Mathematical Psychology, 33, 397-427.

DeKay, M. L., \& Kim, T. G. (2005). When things don’t add up. Psychological Science, 16, 667-672.

Einhorn, H. J., \& Hogarth, R. M. (1985). Ambiguity and uncertainty in probabilistic inference. Psychological Review, 92, 433-461

Einhorn, H. J., \& Hogarth, R. M. (1986). Decision making under ambiguity. Journal of Business, 59, S225-S250.

Ellsberg, D. (1961). Risk ambiguity and the Savage axioms. Quarterly Journal of Economics, 75, 643-649.

Frisch, D., \& Baron, J. (1988). Ambiguity and rationality. Journal of Behavioral Decision Making, 1, 149-157.

Goldsmith, R. W., \& Sahlin, N. (1983). The role of second-order probabilities in decision making. In O. Humphreys, O. Svenson, and A. Vari (Eds.). Analyzing and aiding decision process (pp. 455-467). Amsterdam: North-Holland.

Kahneman D., \& Tversky, A. (1979). Prospect theory: An analysis of decision under risk. Econometrica, 47, 263-291.

Keren, G. (1991). Additional tests of utility theory under unique and repeated conditions. Journal of Behavioral Decision Making, 4, 297-304.

Keren, G., \& Gerritsen, L. E. M. (1999). On the robustness and possible accounts of ambiguity aversion. Acta Psychologica, 103, 149-172. 
Keren, G., \& Wagenaar, W. A. (1987). Violation of utility theory in unique and repeated gambles. Journal of Experimental Psychology: Learning, Memory, and Cognition, 13, 387-391.

Knight, F. H. (1921). Risk, uncertainty and profit. Boston: Houghton, Schaffner \& Marx. Lichtenstein, S., \& Slovic, P. (1971). Reversals of preferences between bids and choices in gambling decisions. Journal of Experimental Psychology, 89, 46-55.

Lopes, L. L. (1981). Decision making in the short run. Journal of Experimental Psychology: Human Learning and Memory, 7, 377-385.

Lopes, L. L. (1996). When time is of the essence: Averaging, aspiration, and the short run. Organizational Behavior and Human Decision Processes, 65, 179-189.

MacCrimmon, K. R., \& Larsson, S. (1979). Utility theory: Axioms versus "paradoxes.” In M. Allais and O. Hagen (Eds.), Expected utility and the Allais paradox (pp. 333-409). Dordrecht: D. Reidel.

Montgomery, H., \& Adelbratt, T. (1982). Gambling decisions and information about expected value. Organizational Behavior and Human Performance, 29, 39-57.

Pulford, B. D., \& Colman, A. M. (2007). Ambiguous games: Evidence for strategic ambiguity aversion. Quarterly Journal of Experimental Psychology, 60, 1083-1100.

Pulford, B. D., \& Colman, A. M. (2008). Size doesn’t really matter: Ambiguity aversion in Ellsberg urns with few balls. Experimental Psychology, 55, 31-37.

Redelmeier, D. A., \& Tversky, A. (1992). On the framing of multiple prospects. Psychological Science, 3, 191-193.

Rode, C., Cosmides, L., Hell, W., \& Tooby, J. (1999). When and why do people avoid unknown probabilities in decisions under uncertainty? Testing some predictions from optimal foraging theory. Cognition, 72, 269-304.

Samuelson, P. A. (1963). Risk and uncertainty: A fallacy of large numbers. Scientia, 98, 108-113.

Slovic, P., \& Lichtenstein, S. (1968). The importance of variance preferences in gambling decisions. Journal of Experimental Psychology, 78, 646-654.

Stokes, M. E., Davis, C. S., \& Koch, G. G. (1995). Categorical data analysis using the SAS System. Cary, NC: SAS Institute Inc.

Thaler R. H., Tversky, A., Kahneman, D., \& Schwartz, A. (1997). The effect of myopia and loss aversion on risk taking: An experimental test. Quarterly Journal of Economics, 112, 647-661.

Tversky, A., \& Bar-Hillel, M. (1983). Risk: The Long and the Short. Journal of Experimental Psychology: Learning, Memory, and Cognition, 9, 713-717.

Tversky A., \& Kahneman, D. (1992). Advances in prospect theory: Cumulative representation under uncertainty. Journal of Risk and Uncertainty, 5, 297-323. 
Tversky, A., Sattath, S., \& Slovic, P. (1988). Contingent weighting in judgment and choice. Psychological Review, 95, 371-384.

Wedell, D. H., \& Böckenholt, U. (1990). Moderation of preference reversals in the long run. Journal of Experimental Psychology: Human Perception and Performance, 16, 429-438.

Wedell, D. H., \& Böckenholt, U. (1994). Contemplating single versus multiple encounters of a risky prospect. American Journal of Psychology, 107, 499-518.

Yates, J. F., \& Zukowski, L. G. (1976). Characterization of ambiguity in decision making. Behavioral Science, 21, 19-25.

\section{Footnote}

${ }^{1} \mathrm{~A}$ third experiment was omitted from this article because it yielded exactly the same results for choices as Experiments 1 and 2. Full details may be obtained from the first author. 